Relations industrielles

Industrial Relations

\title{
Index analytique des articles
}

Volume 26, numéro 4, 1971

URI : https://id.erudit.org/iderudit/028289ar

DOI : https://doi.org/10.7202/028289ar

Aller au sommaire du numéro

Éditeur(s)

Département des relations industrielles de l'Université Laval

\section{ISSN}

0034-379X (imprimé)

1703-8138 (numérique)

Découvrir la revue

Citer ce document

(1971). Index analytique des articles. Relations industrielles / Industrial

Relations, 26(4), 1083-1092. https://doi.org/10.7202/028289ar

Tous droits réservés (C) Département des relations industrielles de l'Université Laval, 1971
Ce document est protégé par la loi sur le droit d'auteur. L’utilisation des services d'Érudit (y compris la reproduction) est assujettie à sa politique d'utilisation que vous pouvez consulter en ligne.

https://apropos.erudit.org/fr/usagers/politique-dutilisation/ 


\section{Travail et main-d'oeuvre - Québec}

Evaluation d'un programme de formation professionnelle mis sur pied par le comité de reclassement des travailleurs de la Coopérative Fédérée du Québec, Filiale Legrade Inc. (Jean Sexton). no 3, p. 592

Le Conseil Consultatif du Travail et de la Main-d'Oeuvre publie son rapport annuel pour 1970-1971. no 3 , p. 773

\section{Index analytique des articles}

\section{Accréditation}

Le caractère majoritaire, principe d'accréditation. La Cour Suprême du Canada réaffirme le droit du syndicat majoritaire à l'accréditation. (Pierre Verge). no 3, p. 764

\section{Action politique}

L'action politique du Congrès du Travail du Canada et de la Fédération Américaine du Travail : une comparaison depuis 1956. (Jean Boivin). no 3 , p. 541

\section{Actionnariat ouvrier}

Bilan de l'année 1970 en droit du travail français : le salaire minimum interprofessionnel garanti ; l'actionnariat ouvrier à la Régie Renault et les salaires en général; la mensualisation des horaires, la formation professionnelle. (G. H. Camerlynck).

no 2 , p. 490

Ancienneté

Les conventions collectives et leurs clauses d'ancienneté. Nature de la convention collective au Canada; examen des clauses d'ancienneté, leur contenu et leur fréquence statistique pour certains secteurs. (Felix Quinet). no 4, p. 890

Arbitrage

Judicial Review of Labour Arbitration in Ontario. Accentuation des conflits entre les arbitres du travail et les tribunaux en Ontario résultant en un nombre croissant d'arrêtés judiciaires cassant les sentences arbitrales. Etude des décisions récentes dans le but de mettre en lumière les tendances de la pensée juridique en Ontario. (Mark Thompson).

no 2, p. 471

L'embauchage d'un salarié lié par une convention collective peut-il être assorti de conditions particulières non contenues dans celle-ci et dans quelle mesure l'arbitre des griefs peut-il tenir compte d'une telle entente particulière. (Pierre Verge).

no 2 , p. 502

\section{Arbitrage obligatoire}

Compulsory Arbitration in British Columbia : Bill 33. Raisons de l'introduction de la loi sur l'arbitrage obligatoire en Colombie-Britannique; analyse des conflits résolus sous cette loi; effet de la loi sur la fréquence et la durée des grèves et son rôle en ce qui concerne l'avenir des relations patronalesouvrières dans cette province. (Peter Z.W. Tsong). no 3, p. 744

Assurance-chômage

Le Livre Blanc sur l'assurance-chômage : appréciation générale et examen de quelques points spécifiques. (Yvon Valcin).

Avantages sociaux

The Economics of Employee Benefits. Le poste des coûts de la main-d'oeuvre communément appelé avantages sociaux représente une part importante du coût global de la main-d'oeuvre dans les frais de production. Facteurs qui servent à déterminer le rapport entre les compléments sociaux et les salaires en espèce dans le coût global de la main-d'oeuvre. (Robert Swidinsky). 


\section{Bureau de Recherches sur les Traitements}

Le rôle de la recherche dans la négociation collective centralisée. Contexte des négociations collectives au sein duquel fonctionne le Bureau de Recherches sur les Traitements. (Felix Quinet).

\section{Cadres}

American Transfer Policies : Costs and Benefits. Coûts et bénéfices des politiques américaines de mutation du personnel de cadre. (William F. Glueck). no 3 , p. 708

L'unité d'accréditation des professionnels. L'article 20 du Code du travail et la syndicalisation des ingénieurs et autres scientifiques. Jugement du Tribunal du Travail dans le cas de la RCA Limited. (Claude D'Aoust).

no 3 , p. 768

Le syndicalisme est-il la réponse aux problèmes des cadres? Modification récente en divers milieux québecois des attitudes traditionnelles à l'endroit des «cadres» d'entreprise. Faiblesse lexicologique du terme «cadres». Esquisse de typologie des cadres. Solutions institutionnelles à la disposition des «cadres 》: action patronale, association de cadres, syndicalisme de cadres et législation élargie. (Roger Chartier).

no 2, p. 399

Centre de Relations Industrielles (France)

Le rôle et les fonctions des Centres de Relations Industrielles en France. Caractéristiques du système de relations industrielles en France, leur structure et leur organisation. Rôle de l'université, recherche, enseignement et formation en ce domaine. (Pierre Candau).

no 3 , p. 726

Centre des Dirigeants d'Entreprises

Eléments d'une stratégie de l'entreprise. Réponse au conflit entre responsabilités économiques et responsabilités sociales : un défi aux dirigeants. (Centre des Dirigeants d'Entreprises).

no 4 , p. 970

\section{Changements technologiques}

An Economic Critique of Current Technological Change Displacement Techniques. Evaluation de l'efficacité des moyens utilisés par les parties contractantes dans le but d'atténuer les effets des changements technologiques pour le travailleur. Il faut considérer ici l'efficacité en fonction des pertes que les travailleurs licenciés peuvent encourir. (David Phillips Ross). no 4, p. 924

\section{Changements technologiques}

Human Adjustment to Technological Change : an Economist's View. Comment la négociation collective a traité le problème du déplacement des travailleurs et quel genre de tendances les changements apportés peuvent inculquer aux relations du travail et à la négociation collective. (Arthur A. Kruger).

no 2 , p. 265

Reopeners : the Task Force on Labour Relations and Freedman. Rôle du secteur privé et du secteur public dans le domaine de la compensation des travailleurs déplacés par les changements technologiques. Analyse de la recommandation du Rapoprt Woods permettant la réouverture de la convention collective pour la négociation des changements technologiques avec considération des propositions du Rapport Freedman. (David P. Ross).

no 1 , p. 213

Some Comments on the Task Force on Labour Relations, Freedman and Reopeners : a Reply to David P. Ross. Commentaires sur l'analyse de David $P$. Ross concernant la réouverture de la convention collective pour: la négociation des changements technologiques. (H. D. Woods). no 1, p. 222

\section{Chômage}

Regional Unemployment Rates and Total Economic Growth in Canada, 19531968. Essai d'évaluation de l'hypothèse que le processus de la croissance économique générale ait un impact différent sur l'emploi selon les cinq régions économiques du Canada. Mesure de la synchronisation de l'impact de la croissance économique générale sur les taux de chômage national et régionaux. (Arthur W. Donner). 


\section{Clauses d'ancienneté}

Les conventions collectives et leurs clauses d'ancienneté. Nature de la convention collective au Canada; examen des clauses d'ancienneté, leur contenu et leur fréquence statistique pour certains secteurs. (Felix Quinet).

no 4 , p. 890

\section{Code du Travail}

Rôle et fonctions de l'enquêteur tels que définis par le Code du Travail. (Claude D'Aoust). no 1, p. 229

\section{Conflits industriels}

Trade-Unions in an Age of Pluralism and Structural Change. Passant en revue le malaise industriel qui a caractérisé l'Europe de l'Ouest et les EtatsUnis au cours des trois dernières années, l'auteur décrit et analyse les nouvelles tendances du mouvement syndical pour s'adapter à cette nouvelle situation. (Solomon Barkin).

no 4 , p. 801

\section{Congrès du Travail du Canada}

L'action politique du Congrès du Travail du Canada et de la Fédération Américaine du Travail: une comparaison depuis 1956. (Jean Boivin).

\section{Conseil Consultatif du Travail et de la Main-d'Oeuvre}

Le Conseil Consultatif du Travail et de la Main-d'Oeuvre publie son rapport annuel pour 1970-1971.

no 3 , p. 773

\section{Convention collective}

L'embauchage d'un salarié lié par une convention collective peut-il être assorti de conditions particulières non contenues dans celle-ci et dans quelle mesure l'arbitre des griefs peut-il tenir compte d'une telle entente particulière. (Pierre Verge).

no 2. p. 502

La violation de la convention collective peut-elle donner ouverture à des dommages-intérêts ? Problème de la détermination de l'instance habilitée à ordonner le paiement de dommages-intérêts. (Pierre Verge). no 1, p. 234

Les conventions collectives et leurs clauses d'ancienneté. Nature de la convention collective au Canada; examen des clauses d'ancienneté, leur contenu et leur fréquence statistique pour certains secteurs. (Felix Quinet). no 4, p. 890

Reopeners: The Task Force on Labour Relations and Freedman. Rôle du secteur privé et du secteur public dans le domaine de la compensation des travailleurs déplacés par les changements technologiques. Analyse de la recommandation du Rapport Woods permettant la réouverture de la convention collective pour la négociation des changements technologiques avec considération des propositions du rapport Freedman. (David P. Ross). no 1, p. 213

Some Comments on the Task Force on Labour Relations, Freedman and Reopeners: a Reply to David P. Ross. Commentaires sur l'analyse de David $P$. Ross concernant la réouverture de la convention collective pour la négociation des changements technologiques. (H. D. Woods). no 1, p. 222

Coopérative Fédérée du Québec

Evaluation d'un programme de formation professionnelle mis sur pied par le Comité de Reclassement des Travailleurs de la Coopérative Fédérée du Québec, Filiale Legrade Inc. (Jean Sexton). no 3, p. 592

\section{Coûts de Production}

The Economics of Employee Benefits. Le poste des coûts de la main-d'oeuvre communément appelé Avantages Sociaux représente une part importante du coût global de la main-d'oeuvre dans les frais de production. Facteurs qui servent à déterminer le rapport entre les compléments sociaux et les salaires en espèce dans le coût global de la main-d'oeuvre. (Robert Swidinsky). no 4 , p. 907 


\section{Croissance économique}

Regional Unemployment Rates and Total Economic Growth in Canada, 19531968. Essai d'évaluation de l'hypothèse que le processus de la croissance économique générale ait un impact différent sur l'emploi selon les cinq régions économiques du Canada. Mesure de la synchronisation de l'impact de la croissance économique générale sur les taux de chômage national et régionaux. (Arthur W. Donner). no 3 , p. 721

\section{Dommages-intérêts}

$\mathrm{La}$ violation de la convention collective peut-elle donner ouverture à des dommages-intérêts ? Problème de la détermination de l'instance habilitée à ordonner le paiement de dommages-intérêts. (Pierre Verge). no 1, p. 234

Droit du Travail

Bilan de l'année 1970 en Droit du Travail français : le salaire minimum interprofessionnel garanti; l'actionnariat ouvrier à la Régie Renault et les salaires en général ; la mensualisation des horaires, la formation professionnelle. (G. H. Camerlynck). no 2, p. 490

\section{Eclairage}

Problèmes posés dans l'entreprise par l'éclairage des locaux. (Pierrette Sartin). no 4 , p. 951

\section{Education}

Decentralized Bargaining: Its Problems and Directions in the Public Education Systems of Ontario and the Western Provinces. Problèmes et tendance de la négociation collective décentralisée dans les systèmes d'éducation publique de l'Ontario, Alberta et Colombie-Britannique. (J. Douglas Muir). no 1, p. 124

\section{Embauchage}

L'embauchage d'un salarié lié par une convention collective peut-il être assorti de conditions particulières non contenues dans celle-ci et dans quelle mesure l'arbitre des griefs peut-il tenir compte d'une telle entente particulière. (Pierre Verge).

\section{Emploi}

Regional Unemployment Rates and Total Economic Growth in Canada, 19531968. Essai d'évaluation de l'hypothèse que le processus de la croissance économique générale ait un impact différent sur l'emploi selon les cinq régions économiques du Canada. Mesure de la synchronisation de l'impact de la croissance économique générale sur les taux de chômage national et régionaux. (Arthur W. Donner). no 3 , p. 721

\section{Emplois disponibles}

Job Vacancy Survey as a Tool of Labour Market Information in Developing Countries. Présentation d'une méthode pour inventorier les emplois disponibles dans un contexte de roulement de main-d'oeuvre. (Ozay Mehmet). no 3, p. 692

\section{Enquêteur}

Rôle et fonctions de l'enquêteur tels que définis par le Code du travail. (Claude D'Aoust).

\section{Entreprise}

Eléments d'une stratégie de l'entreprise. Réponse au conflit entre responsabilités économiques et responsabilités sociales: un défi aux dirigeants. (Centre des Dirigeants d'Entreprises).

\section{Entreprises multinationales}

The Multinational Corporation and Industrial Relations: the European Approach. Problème d'adaptation posé par l'expansion rapide des entreprises multinationales aux divers systèmes de relations industrielles en Europe ces vingtcinq dernières années. Comment le mouvement ouvrir a réagi à cette situation. (Paul Malles). 
Fédération Américaine du Travail

L'action politique du Congrès du Travail du Canada et de la Fédération américaine du Travail : une comparaison depuis 1956. (Jean Boivin). no 3, p. 541

Fonction Publique du Canada

Le rôle de la recherche dans la négociation collective centralisée. Contexte des négociations collectives au sein duquel fonctionne le Bureau de Recherches sur les Traitements. (Felix Quinet). no 1, p. 184

Formation des Adultes

A Countercyclical Training Programme for Canada ? Rôle potentiel de la politique de la main-d'oeuvre en général, et des programmes de formation des adultes en particulier, en tant qu'instruments de stabilisation économique. (Keith Newton).

no 4 , p. 865

Formation professionnelle

Bilan de l'année 1970 en Droit du Travail français : le salaire minimum interprofessionnel garanti ; l'actionnariat ouvrier à la Régie Renault et les salaires en général; la mensualisation des horaires, la formation professionnelle. (G. H. Camerlynck). no 2 , p. 490

Evaluation d'un programme de formation professionnelle mis sur pied par le Comité de Reclassement des Travailleurs de la Coopérative Fédérée du Québec, Filiale Legrade Inc. (Jean Sexton). no 3, p. 592

Grève

Compulsory Arbitration in British Columbia: Bill 33. Raisons de l'introduction de la loi sur l'arbitrage obligatoire en Colombie-Britannique ; analyse des conflits résolus sous cette loi ; effet de la loi sur la fréquence et la durée des grèves et son rôle en ce qui concerne l'avenir des relations patronales-ouvrières dans cette province. (Peter Z.W. Tsong).

no 3, p. 744

\section{Hôpitaux}

Labor Relations in Ontario Hospitals : a Question of Survival. Les événements survenus ces dix dernières années font douter de l'existence de réelles relations du travail dans les hôpitalx d'Ontario. Conditions de la survie effective des relations du travail dans ce secteur. (Fraser Isbester et Sandra Castle). no 2, p. 345

\section{Hygiène du Travail}

Problèmes posés dans l'entreprise par l'éclairage des locaux. (Pierrette Sartin). no 4 , p. 951

\section{Industrie de la Construction}

Investment-Wages: Theory and Application. Définition et fondements du salaire d'investissement ; principes de l'économie sociale de marché ; exemple du salaire d'investissement dans l'industrie de la construction Ouest-Allemande. (Gerhard Reber).

no 2, p. 363

La négociation sectorielle par décision de l'Etat: le cas de la construction au Québec. Principaux traits de la «Loi des Relations de Travail dans l'Industrie de la Construction ». Régime légal et situation; objectifs et implications; applications et problèmes. (Gérard Hébert). no 1, p. 84

Industrie des Matériaux de Construction

Multi-Employer Bargaining: the Case of B.C. Coast Lumber Industry. Particularités de la négociation multi-employeur dans une industrie dite primaire. Analyse de la situation dans l'industrie des matériaux de construction de la Colombie-Britannique caractérisée par de fréquents conflits industriels. (Stuart Jamieson). no 1, p. 146

\section{Industrie du Camionnage}

Multi-Employer Bargaining - Ontario Trucking : a Case Study. Avantages et désavantages de la négociation multi-employeur dans l'industrie Ontarienne du camionnage. (Graeme H. McKechnie). 
Industrie du Fer et de l'Acier

Collective Bargaining and Wage Equalization in Canada's Iron and Steel Industry, 1939-1964. Détermination du degré selon lequel l'égalité des salaires est présente dans l'Industrie Canadienne du Fer et de l'Acier et identification des influences qui ont contribué à ces tendances égalisantes. (Bryan C. Williams). no 2, p. 308

\section{Ingénieurs}

L'unité d'accréditation des professionnels. L'article 20 du Code du travail et la syndicalisation des ingénieurs et autres scientifiques. Jugement du Tribunal du Travail dans le cas de la RCA Limited. (Claude D'Aoust). no 3, p. 768 The Labor Market Experience of Engineers in North America. Etude comparative des expériences vécues par les ingénieurs canadiens et américains sur les divers marchés du travail. (Archibald D. Boyd, Andrew C. Gross, Raymond R. McKay).

no 4, p. 829

Langue de Travail

L'unité de négociation appropriée et la langue de travail: l'affaire de la Régionale Le Royer. (Claude D'Aoust). no 2, p. 497

Législation Canadienne du Travail

Chronologie de la Législation Canadienne en matière de relations du travail. (1868-1971).

no 4 , p. 1013

\section{Licenciements}

An Economic Critique of Current Technological Change Displacement Techniques. Evaluation de l'efficacité des moyens utilisés par les parties contractantes dans le but d'atténuer les effets des changements technologiques pour le travailleur. Il faut considérer ici l'efficacité en fonction des pertes que les travailleurs licenciés peuvent encourir. (David Phillips Ross). no 4, p. 924

Loi des Relations de Travail dans l'Industrie de la Construction La négociation sectorielle par décision de l'Etat : le cas de la construction au Québec. Principaux traits de la «Loi des Relations de Travail dans l'Industrie de la Construction ». Régime légal et situation; objectifs et implications; applications et problèmes. (Gérard Hébert). no 1, p. 84

Loi des Relations Ouvrières de l'Ontario

Reform of the Status Quo Ontario's Bill 167. Amendements faits en 1970 à la Loi des relations ouvrières de l'Ontario; leurs conséquences et commentaires sur les omissions sérieuses de la loi. (John Crispo). no 4 , p. 852

Loi sur les Relations Industrielles (Grande-Bretagne)

The British Industrial Relations Bill: an Analysis. Analyse de la nouvelle loi britannique sur les relations industrielles adoptée en décembre 1970. (T.G. Whittingham et $\mathrm{B}$. Towers).

no 3 , p. 620

Main-d'oeuvre

Job Vacancy Survey as a Tool of Labour Market Information in Developing Countries. Présentation d'une méthode pour inventorier les emplois disponibles dans un contexte de roulement de main-d'oeuvre. (Ozay Mehmet). no 3, p. 692

The Economics of Employee Benefits. Le poste des coûts de la main-d'oeuvre communément appelé Avantages Sociaux représente une part importante du coût global de la main-d'oeuvre dans les frais de production. Facteurs qui servent à déterminer le rapport entre les compléments sociaux et les salaires en espèce dans le coût global de la main-d'oeuvre. (Robert Swidinsky). no 4 , p. 907

\section{Marché du Travail}

Job Vacancy Survey as a Tool of Labour Market Information in Developing Countries. Présentation d'une méthode pour inventorier les emplois disponibles dans un contexte de roulement de main-d'oeuvre. (Ozay Mehmet). no 3, p. 692 
The Labor Market Experience of Engineers in North America. Etude comparative des expériences vécues par les ingénieurs canadiens et américains sur les divers marchés du travail. (Archibald D. Boyd, Andrew C. Gross, Raymond R. McKay). no 4 , p. 829

\section{Mobilité de la Main-d'oeuvre}

Labor Mobility : An Investment in Human Capital Approach. Explication des facteurs économiques sous-jacents à la mobilité du travail, qu'elle soit géographique, professionnelle ou industrielle. (Athenas P. Kottis). no 4, p. 943

Mutation du Personnel de Cadre

American Transfer Policies : Costs and Benefits. Coûts et bénéfices des politiques américaines de mutation du personnel de cadre. (William F. Glueck). no 3, p. 708

\section{Négociation collective}

British Collective Bargaining : the Challenge of the 1970's. Impact de la réforme du système de relations industrielles britannique qui eut lieu dans les années 1960. Défis posés à la négociation collective en Angleterre pour les années 1970. (Robert F. Banks). no 3, p. 642

Collective Bargaining and Wage Equalization in Canada's Iron and Steel Industry, 1939-1964. Détermination du degré selon lequel l'égalité des salaires est présente dans l'Industrie Canadienne du Fer et de l'Acier et identification des influences qui ont contribué à ces tendances égalisantes. (Bryan C. Williams). no 2, p. 308

Decentralized Bargaining: Its Problems and Directions in the Public Education Systems of Ontario and the Western Provinces. Problèmes et tendances de la négociation collective décentralisée dans les systèmes d'éducation publique de l'Ontario, Alberta et Colombie-Britannique. (J. Douglas Muir). no 1, p. 124

Human Adjustment to Technological Change : an Economist's View. Comment la négociation collective a traité le problème du déplacement des travailleurs et quel genre de tendances les changements apportés peuvent inculquer aux relations du travail et à la négociation collective. (Arthur A. Kruger).

no 2 , p. 265

Le rôle de la recherche dans la négociation collective centralisée. Contexte des négociations collectives au sein duquel fonctionne le Bureau de recherches sur les Traitements. (Felix Quinet).

no 1, p. 184

Reopeners: The Task Force on Labour Relations and Freedman. Rôle du secteur privé et du secteur public dans le domaine de la compensation des travailleurs déplacés par les changements technologiques. Analyse de la recommandation du Rapport Woods permettant la réouverture de la convention collective pour la négociation des changements technologiques avec considération des propositions du Rapport Freedman. (David P. Ross). no 1, p. 213 Some Comments on the Task Force on Labour Relations, Freedmand and Reopeners : a Reply to David P. Ross. Commentaires sur l'analyse de David P. Ross concernant la réouverture de la convention collective pour la négociation des changements technologiques. (H. D. Woods). no 1, p. 222

Négociation collective bi-nationale

Centralized Collective Bargaining: U.S.-Canada Experience. Examen de la négociation collective bi-nationale impliquant une délégation de prise de décision vers les Etats-Unis, soit en suivant les normes américaines, soit en déléguant effectivement la prise de décision à des cadres américains. Sources, tactiques, stratégies et implications des conventions bi-nationales. (Bryan $\mathbf{M}$. Downie). no 1, p. 38

\section{Négociation collective multi-employeur}

Multi-Employer Bargaining - Ontario Trucking : a Case Study. Avantages et désavantages de la négociation multi-employeur dans l'industrie ontarienne du camionnage. (Graeme H. McKechnie). 
Multi-Employer Bargaining: the Case of B.C. Coast Lumber Industry. Particularités de la négociation multi-employeur dans une industrie dite primaire. Analyse de la situation dans l'industrie des matériaux de construction de la Colombie-Britannique caractérisée par de fréquents conflits industriels. (Stuart Jamieson). no 1 , p. 146

Négociation collective sectorielle

La négociation collective sectorielle : définition, difficultés et conditions de succès. (Robert Sauvé). no 1 , p. 3

La négociation sectorielle par décision de l'Etat: le cas de la construction au Québec. Principaux traits de la «Loi des Relations de Travail dans l'Industrie de la Construction ». Régime légal et situation; objectifs et implications; applications et problèmes. (Gérard Hébert).

no 1, p. 84

La négociation sectorielle : une panacée ? Commentaire de l'article de Normand Cinq-Mars paru dans «Relations Industrielles», volume 25, no 3. (Bernard Brody).

no 2, p. 429

\section{Pays en voie de Développement}

Extension and Feasibility of the Woods Report to the Developing Countries. Evaluation du modèle théorique utilisé dans le Rapport Woods; possibilités de son application pour l'analyse comparative des systèmes de relations industrielles; possibilités de l'exportation du système de relations industrielles canadien dans les pays en voie de développement. (Syed M. A. Hameed).

no 3 , p. 575

Job Vacancy Survey as a Tool of Labour Marquet Information in Developing Countries. Présentation d'une méthode pour inventorier les emplois disponibles dans un contexte de roulement de main-d'oeuvre. (Ozay Mehmet). no 3, p. 692

Pluralisme syndical

Trade-Unions in an Age of Pluralism and Structural Change. Passant en revue le malaise industriel qui a caractérisé l'Europe de l'Ouest et les Etats-Unis au cours des trois dernières années, l'auteur décrit et analyse les nouvelles tendances du mouvement syndical pour s'adapter à cette nouvelle situation. (Solomon Barkin).

no 4 , p. 801

\section{Politique de Main-d'oeuvre}

A Countercyclical Training Programme for Canada? Rôle potentiel de la politique de la main-d'oeuvre en général, et des programmes de formation des adultes en particuliers, en tant qu'instruments de stabilisation économique. (Keith Newton).

no 4, p. 865

\section{Rapport Freedman}

Reopeners: The Task Force on Labour Relations and Freedman. Rôle du secteur privé et du secteur public dans le domaine de la compensation des travailleurs déplacés par les changements technologiques. Analyse de la recommandation du Rapport Woods permettant la réouverture de la convention collective pour la négociation des changements technologiques avec considération des propositions du Rapport Freedman. (David P. Ross). no 1, p. 213

Some Comments on the Task Force on Labour Relations, Freedmand and Reopeners : a Reply to David P. Ross. Commentaires sur l'analyse de David $P$. Ross concernant la réouverture de la convention collective pour la négociation des changements technologiques. (H. D. Woods). no 1, p. 222

\section{Rapport Woods}

Extension and Feasibility of the Woods Report to the Developing Countries. Evaluation du modèle théorique utilisé dans le Rapport Woods; possibilités de son application pour l'analyse comparative des systèmes de relations industrielles possibilités de l'exportation du système de relations industrielles canadien dans les pays en voie de développement. (Syed M. A. Hameed). 
Reopeners: The Task Force on Labour Relations and Freedman. Rôle du secteur privé et du secteur public dans le domaine de la compensation des travailleurs déplacés par les changements technologiques. Analyse de la recommandation du Rapport Woods permettant la réouverture de la convention collective pour la négociation des changements technologiques avec considération des propositions du Rapport Freedman. (David P. Ross).

no 1 , p. 213

Some Comments on the Task Force on Labour Relations, Freedman and Reopeners : a Reply to David P. Ross. Commentaires sur l'analyse de David P. Ross concernant la réouverture de la convention collective pour la négociation des changements technologiques. (H. D. Woods).

no 1, p. 222

Recherche

Le rôle de la recherche dans la négociation collective centralisée. Contexte des négociations collectives au sein duquel fonctionne le Bureau de Recherches sur les Traitements. (Felix Quinet).

no 1, p. 184

Reclassement des Travailleurs

Evaluation d'un programme de formation professionnelle mis sur pied par le Comité de Reclassement des Travailleurs de la Coopérative Fédérée du Québec, Filiale Legrade Inc. (Jean Sexton). no 3 , p. 592

Régionale Le Royer

L'unité de négociation appropriée et la langue de travail: l'affaire de la Régionale Le Royer. (Claude D'Aoust). no 2, p. 497

Responsabilités de l'Entreprise

Eléments d'une stratégie de l'entreprise. Réponse au conflit entre responsabilités économiques et responsabilités sociales : un défi aux dirigeants. (Centre des Dirigeants d'Entreprises). no 4 , p. 970

Salaire d'Investissement

Investment-Wages: Theory and Application. Définition et fondements du salaire d'investissement; Principes de l'Economie Sociale de Marché; Exemple du Salaire d'Investissement dans l'Industrie de la Construction Ouest-Allemande. (Gerhard Reber).

no 2, p. 363

Salaire minimum

Bilan de l'année 1970 en Droit du Travail Français; Le salaire minimum Interprofessionnel garanti ; L'actionnariat ouvrier à la Régie Renault et les salaires en général ; La mensualisation des horaires, la formation professionnelle. (G. H. Camerlynck). no 2 , p. 490

Salaires

Collective Bargaining and Wage Equalization in Canada's Iron and Steel Industry, 1939-1964. Détermination du degré selon lequel l'égalité des salaires est présenté dans l'Industrie Canadienne du Fer et de l'Acier et identification des influences qui ont contribué à ces tendances égalisantes. (Bryan C. Williams).

no. 2 , p. 308

The Economics of Employee Benefits. Le poste des coûts de la main-d'oeuvre communément appelé Avantages Sociaux représente une part importante du coût global de la main-d'oeuvre dans les frais de production. Facteurs qui servent à déterminer le rapport entre les compléments sociaux et les salaires en espèce dans le coût global de la main-d'oeuvre. (Robert Swidinski).

no 4 , p. 907

\section{Stabilisation économique}

A Countercyclical Training Programme for Canada? Rôle potentiel de la politique de la main-d'oeuvre en général, et des programmes de formation des adultes en particulier, en tant qu'instruments de stabilisation économique. (Keith Newton). no 4 , p. 865

Syndicalisme américain

L'action politique du Congrès du Travail du Canada et de la Fédération Américaine du Travail : Une comparaison depuis 1956. (Jean Boivin). 
Trade-Unions in an Age of Pluralism and Structural Change. Passant en revue le malaise industriel qui a caractérisé l'Europe de l'Ouest et les Etats-Unis au cours des trois dernières années, l'auteur décrit et analyse les nouvelles tendances du Mouvement Syndical pour s'adapter à cette nouvelle situation. (Solomon Barkin). no 4 , p. 801

\section{Syndicalisme canadien}

L'action politique du Congrès du Travail et de la Fédération Américaine du Travail : Une comparaison depuis 1956. (Jean Boivin). no 3, p. 541

\section{Syndicalisme de Cadres}

L'unité d'accréditation des professionnels. L'article 20 du Code du travail et la syndicalisation des ingénieurs et autres scientifiques. Jugement du Tribunal du Travail dans le cas de la RCA Limited. (Claude D'Aoust).

no 3 , p. 768

Le syndicalisme est-il la réponse aux problèmes des cadres? Modification récente en divers milieux québécois des attitudes traditionnelles à l'endroit des «Cadres» d'entreprise. Faiblesse lexicologique du terme «Cadres》. Esquisse de typologie des cadres. Solutions institutionnelles à la disposition des $\ll \mathrm{Ca}-$ dres »: action patronale, association de cadres, syndicalisme de cadres et législation élargie. (Roger Chartier).

no 2, p. 399

\section{Syndicalisme européen}

Trade-Unions in an Age of Pluralism and Structural Change. Passant en revue le malaise industriel qui a caractérisé l'Europe de l'Ouest et les Etats-Unis au cours des trois dernières années, l'auteur décrit et analyse les nouvelles tendances du mouvement syndical pour s'adapter à cette nouvelle situation. (Solomon Barkin).

$$
\text { no } 4 \text {, p. } 801
$$

\section{Systèmes de Relations Industrielles}

British Collective Bargaining: The Challenge of the 1970's. Impact de la réforme du système de relations industrielles Britannique qui eut lieu dans les années 1960. Défis posés à la négociation collective en Angleterre pour les années 1970. (Robert F. Banks). no 3 , p. 642

Extension and Feasibility of the Woods Report to the Developing Countries. Evaluation du modèle théorique utilisé dans le Rapport Woods; possibilités de son application pour l'analyse comparative des systèmes de relations industrielles; possibilités de l'exportation du système de relations industrielles canadien dans les pays en voie de développement. (Syed M. A. Hameed). no 3 , p. 575

Le rôle et les fonctions des Centres de Relations Industrielles en France. Caractéristiques du système de relations industrielles en France, leur structure et leur organisation. Rôle de l'Université, recheche, enseignement et formation en ce domaine. (Pierre Candau).

no 3, p. 726

The Multinational Corporation and Industrial Relations: The European Approach. Problème d'adaptation posé par l'expansion rapide des entreprises multi-nationales aux divers systèmes de relations industrielles en Europe ces vingt-cinq dernières années. Comment le mouvement ouvrier a réagi à cette situation. (Paul Malles).

no 1, p. 64

\section{Tribunaux du Travail}

Judicial Review of Labour Arbritration in Ontario. Accentuation des conflits entre les arbitres du travail et les tribunaux en Ontario résultant en un nombre croissant d'arrêtés judiciaires cassant les sentences arbitrales. Etude des décisions récentes dans le but de mettre en lumière les tendances de la pensée juridique en Ontario. (Mark Thompson).

\section{Unité de Négociation}

L'unité de négociation appropriée et la langue de travail: L'Affaire de la Régionale Le Royer. (Claude D'Aoust). 